\title{
A Bio-inspired Sighted Robot Chases like a Hoverfly
}

\author{
Fabien Colonnier ${ }^{1,2}$, Soledad Ramirez-Martinez ${ }^{1,3}$, Stéphane \\ Viollet $^{1}$, and Franck Ruffier ${ }^{1}$ \\ 1 Aix Marseille Univ, CNRS, ISM, Marseille, France \\ 2 Temasek Labs, National University of Singapore, Singapore, Singapore \\ ${ }^{3}$ Facultad de Ciencias de la Electrónica, Benemérita Universidad Autónoma de \\ Puebla (BUAP), Puebla, Mexico \\ E-mail: fabien.colonnier@nus.edu.sg, soledad.ramirez@correo.buap.mx, \\ stephane.viollet@univ-amu.fr, franck.ruffier@univ-amu.fr
}

August 2018

\begin{abstract}
Here we present a novel bio-inspired visual processing system, which enables a robot to locate and follow a target, using an artificial compound eye called CurvACE. This visual sensor actively scanned the environment at an imposed frequency $(50 \mathrm{~Hz})$ with an angular scanning amplitude of $4.2^{\circ}$ and succeeded in locating a textured cylindrical target with hyperacuity, i.e. much finer resolution than the coarse inter-receptor angle of the compound eye. Equipped with this small, lightweight visual scanning sensor, a Mecanum-wheeled mobile robot named ACEbot was able to follow a target at a constant distance by localizing the right and left edges of the target. The localization of the target's contrasted edges is based on a bio-inspired summation of Gaussian receptive fields in the visual system. By means of its autoadaptive pixels, ACEbot consistently achieved similar pursuit performances under various lighting conditions with a high level of repeatability. The robotic pursuit pattern mimics finely the behavior of the male fly Syritta Pipens L. while pursuing the female. The high similarity in the trajectories as well as the biomimicry of the visual system provides strong support for the hypothesis that flies do maintain center the target and constant its subtended angle during smooth pursuit. Moreover, we discuss the fact that such simple strategy can also provide a trajectory compatible with motion camouflage.
\end{abstract}

\section{Introduction}

Highly efficient target tracking behaviors have been observed throughout the animal kingdom [1]. Particularly, small insects, despite their limited sensory system, display impressive capabilities like catching preys or mates, in order to survive in their ecological environment. For example, the housefly (Fannia canicularis) tracks the female by consistently flying towards it, making most likely no assumptions about its quarry's future position [2]. Likewise, Syritta pipiens L. hoverflies follow their potential mates, keeping the same distance away before trying to catch them. Depending on the position of the female in its field of view, the male will perform either body saccades and sway 
movements when the target is located on the side, or adopt a smooth pursuit strategy [3] when the female is upfront. The male Lucilia can also follow its target for a while before deciding whether it will be a suitable mate [4]. Based on field observations, these Lucilia flies seem to use the size of their target in their Field-Of-View (FOV) and hold its position on the frontal midline of the head. Others, like the robber fly (Holcocephala fusca), chase their target with a constant bearing angle [5]. More complex chasing strategies have also been observed in hoverflies Eristallis and Volucella [6] or in the dragonflies [7], where it seems that a prediction of the target trajectory is involved [8].

The studies of the dragonfly visual system taught us that specific neurons called Elementary Small Target Motion Detectors (ESTMDs) are implicated in the target detection and tracking [9, 10]. A bio-inspired algorithm derived from these observations was shown to be efficient and accurate [11] compared to the state of the art in computer vision [12] and applicable in a robotic context [13]. Another bio-inspired example of target tracking on a mobile robot has been made using an Event-based camera [14].

In this study, we focused on a pursuit scenario in which a holonomic mobile robot (the pursuer) tracks a mobile target, maintaining its visual contact with the target at all times and keeping a constant distance. It was assumed that the pursuer knows the size of the target and that the speed of the target is bounded, as hypothesized by Collett and Land [6. Previous authors have tackled target following challenges using an omnidirectional camera without any communication between the pursuer and the target, and by planning a suitable trajectory for the pursuer [15, 16, 17]. Follow-the-leader scenarios can be said to be a particular case of target tracking, as the leader is identified and can provide the follower(s) with relevant information. Some authors of robotic studies have used infrared (IR) beacons [18, 19, 20, 21], visual pattern recognition processes [22] based on the open-source code Whycon [23], and acoustic signals either alone [24] or combined with radio signals to estimate the distance from a moving target [25]. Stereo vision was also used on a UAV to intercept a moving colored target [26]. On the control point of view, pursuit has been largely studied for missile guidance with Line-Of-Sight techniques [27]. Some elaborated controllers have been designed to be robust to unknown target acceleration and more energy efficient [21], or optimize the time to capture [28], whereas others took the visual sensor FOV into account [29]. Motion camouflage is also a complex pursuit strategy, which aims to make the pursuer either static or producing an optic flow compatible with a fixed object onto the target retina. It has been suggested to be biologically relevant [30] and observed on the dragonfly [31. Several theoretical studies have proposed algorithms to perform such behavior [32, 33, 34, 35].

The pursuit scenario is here achieved by using a bio-inspired artificial compound eye called CurvACE featuring an optical resolution of only a few degrees imposed by the angle between two adjacent artificial ommatidia (photoreceptor + lenslet) [36]. The relatively coarse optical resolution and its low sensitivity (see fig. 15 in [37]) was greatly improved by applying small periodic mechanical vibrations to the whole eye, which resulted in a visual micro-scanning of the environment. Many visual sensors based on active retinal micro-movements (see [38] for a review) have been used for 
various robotic purposes, such as enhancing edge detection [39, 40] and improving obstacle avoidance [41]. Recent studies featuring hyperacuity, i.e. "locating features with a greater accuracy than that corresponding to the resolution imposed by the photoreceptor's limited pitch" as defined by [42], have focused on the processing of the amplitude of the photosensor's output signals to locate an edge/bar using only 2 pixels [43], to locate and track a white cross using complex calibration process [44] or to measure robot 1D egomotion [45]. The visual sensor is a vibrating artificial compound eye called Active CurvACE (i.e. the scanning version of the CurvACE sensor) : Active CurvACE was developed to locate features with hyperacuity [45]. The CurvACE sensor is endowed with bio-inspired micro-scanning movements imposed by a tiny motor and an eccentric mechanism [46, 45]. The Active CurvACE sensor is characterized by 4 specific bio-inspired principles:

- local light adaptation at the pixel level,

- a similar inter-ommatidial angle $\Delta \varphi$ to that observed from the fruitfly's eye,

- a Gaussian shaped angular sensitivity function at the level of each artificial ommatidium,

- a micro-scanning movement with its visual signal processing providing hyperacuity.

In addition, a new algorithm is introduced, inspired by the fusion of angular Gaussian receptive fields observed by Heiligenberg on the electric fish [47], to aggregate the visual signals of the artificial ommatidia. The algorithm takes advantage of the angular Gaussian response of the sensors to improve the linearity of the angular position of the target placed in the FOV of the Active CurvACE sensor. This new linear characteristic was used to make a pursuer robot follow a target. Then, we established that the pursuer equipped with a bio-inspired non-emissive visual sensor was capable of achieving smooth pursuit when following a mobile cylindrical target at a constant distance. Section 2 presents the experimental setup and the objectives. Section 3 describes the bio-inspired visual processing, including a receptive fields summation method used to locate the cylindrical target over a large angular position range within the horizontal FOV of Active CurvACE. Section 4 describes the control system and the kinematics of the pursuer robot. Section 5 shows the performance of the pursuit obtained under 3 different lighting conditions, the repeatability and the comparison with hoverfly trajectories with a discussion on the motion camouflage. Section 6 draws some conclusions and suggests some perspectives.

\section{Experimental setup and objectives}

The pursuer equipped with a bio-inspired visual sensor using only 23 artificial ommatidia was able to perform smooth pursuits when following another mobile robot (the cylindrical target) at a constant distance. The FOV covered by the 23 photosensors was about $97^{\circ}$. As shown in figure 1 a, the two robots were used to test the pursuit scenario: 

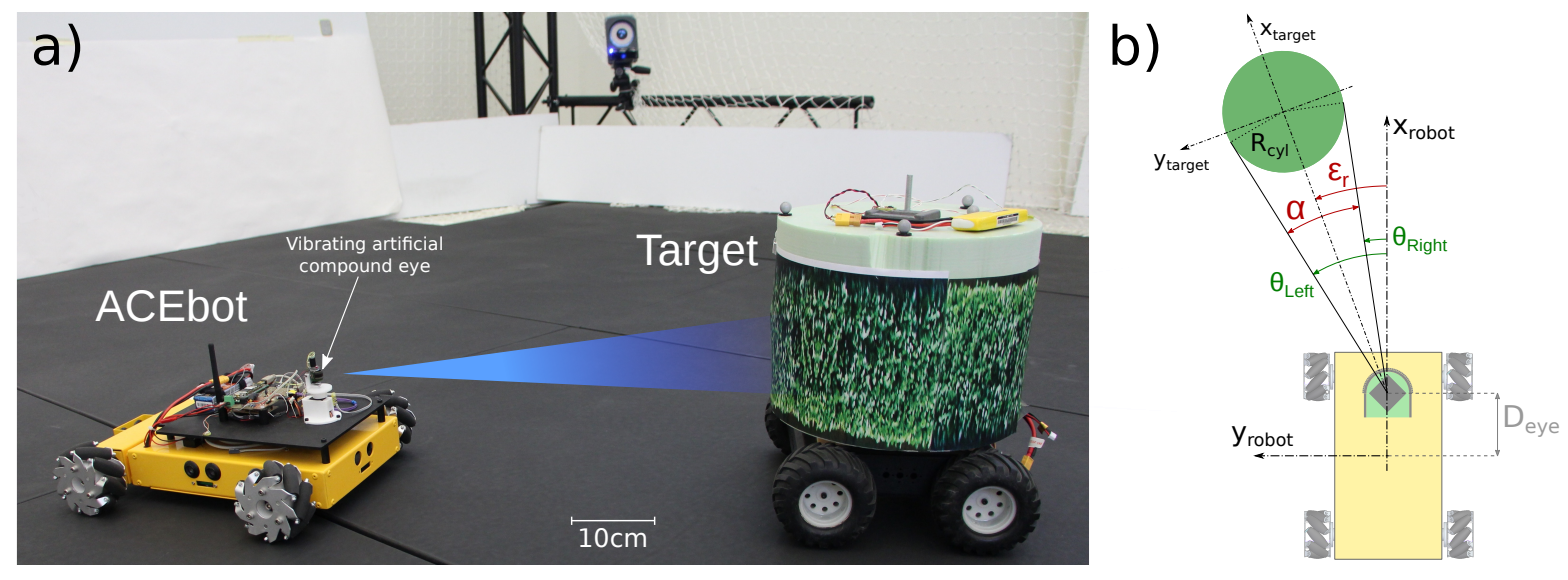

Figure 1. a) An experimental situation where the pursuer (i.e., the yellow robot ACEbot) is following the target (a textured cylinder mounted on a moving wheeled rover) at a constant distance. Thanks to its hyperacute visual sensor, the robot ACEbot is able to lock its heading onto the target and keep a constant distance from the target. b) Scheme of the various parameters and angles used in the control strategy implemented here (see equation (5)).

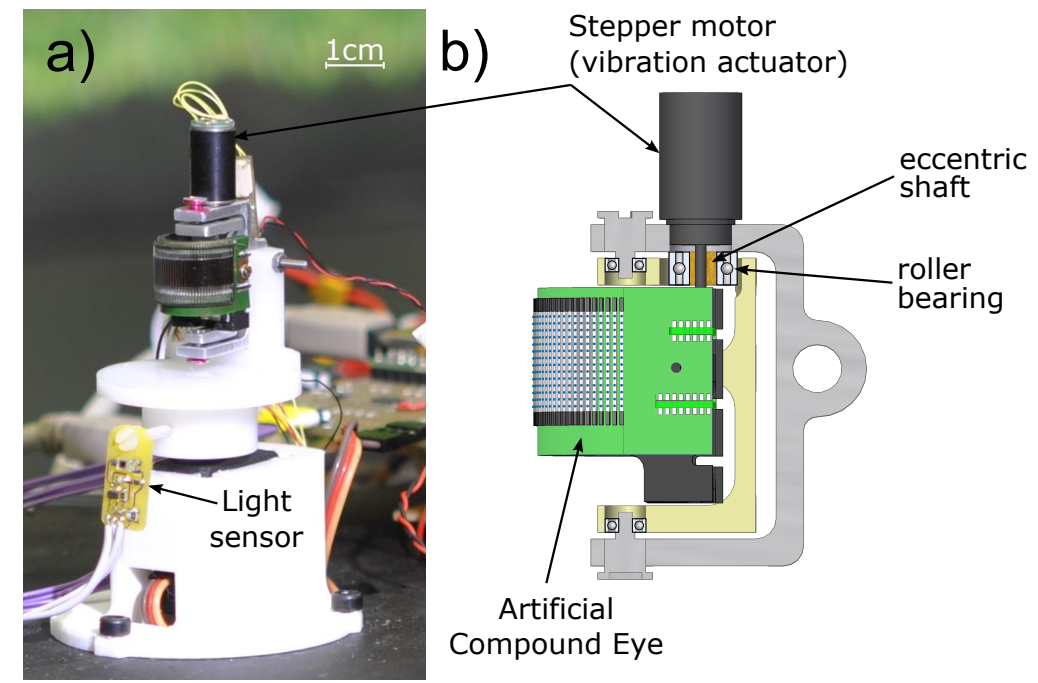

Figure 2. a) Picture of the ACEbot's visual sensor called Active CurvACE and the photodiode used for the illuminance measurements. b) Diagram of the mechanical system, the eccentric shaft is used with a roller bearing to limit the wear in the slotted hole. This Active CurvACE assembling is very compact $(40 \times 27 \times 15 \mathrm{~mm})$ and light (12.5 grams).

- The pursuer, named ACEbot, which stands for "Active Compound Eye on a robot", was a yellow rover equipped with a vibrating artificial compound eye, called Active CurvACE visual sensor (see figure 2), and Mecanum wheels (see figures 1 and E1).

- The target was a non-holonomic rover carrying a textured cylinder (see figure 1a). The two contrasting edges of the cylinder with respect to the background were the two visual cues used by the sighted pursuer to track the target's movement. 
As depicted in figure $1 \mathrm{~b}$, the following notation is used throughout this paper:

- $\theta_{\text {Left }}, \theta_{\text {Right }}$ are the angular position of each edge in the eye's FOV,

- $\epsilon_{r}$ is the retinal error, i.e. the position of the target in the eye's FOV,

- $\alpha$ is the subtended angle, i.e. the size of the target in the FOV,

- $R_{c y l}$ is the radius of the cylinder.

\section{Bio-inspired receptive fields summation to localize a target with hyperacuity}

\subsection{From modulated visual signals to Gaussian-like responses}

The first step of the visual processing is to perform a demodulation of the artificial ommatidia signals which are submitted to a $50 \mathrm{~Hz}$ scanning. It should be noticed that though bio-inspired, the sensor scanning is made at a higher frequency than the one observed on the fly (between $5-7 \mathrm{~Hz}$, see [38] for a review). Figure 3 a details the demodulation strategy. First, a peak filter centered on the scanning frequency extracts the modulated signal, removing the constant value and some of the noise. The absolute value allow to double the frequency, to facilitate the envelope detection. Then, an envelope detector allow a first low-pass filtering without delay and finally a low-pass filter smooth out the demodulated signal.

Compared to [45], the modification of the processing were the addition of the envelope detector and modified filters. All these improvements enabled to increase the dynamic response of the demodulation. Indeed, the cutoff frequency of the last low-pass filter have been increased (from 5 to $20 \mathrm{~Hz}$ ), thus increasing the bandwidth of the visual processing.

To test the responses of the visual processing algorithm, the artificial eye was rotated back and forth in front of the target by activating the visual micro-scanning movements. As shown in figure 3c, the "Edge receptive field" of each pixel's output signal, denoted $P h_{D}$, can be approximated with a Gaussian-like function centered at the angular position of the contrasting edge with respect to the eye (the gaze).

\subsection{From Gaussian-like responses to angular position measurement within the triplet's} FOV

3.2.1. Localization using Gaussian receptive fields Heiligenberg [47] has established that the Weighted Sum (WS) of an array of overlapped Gaussian receptive fields in an electric fish can provide a linear response to a stimulus position. $W S$ computations on an array of sensors with a Gaussian shaped response give an approximation of a straight line $(W S(x, \sigma) \approx \sqrt{\pi} \sigma x$, with $x$ being the stimulus position). Appendix C.1 provides the mathematical definition of the $W S$ and further details about the computation in the case of perfect Gaussian receptive fields. The validity of this linear approximation was proved in [48]. 


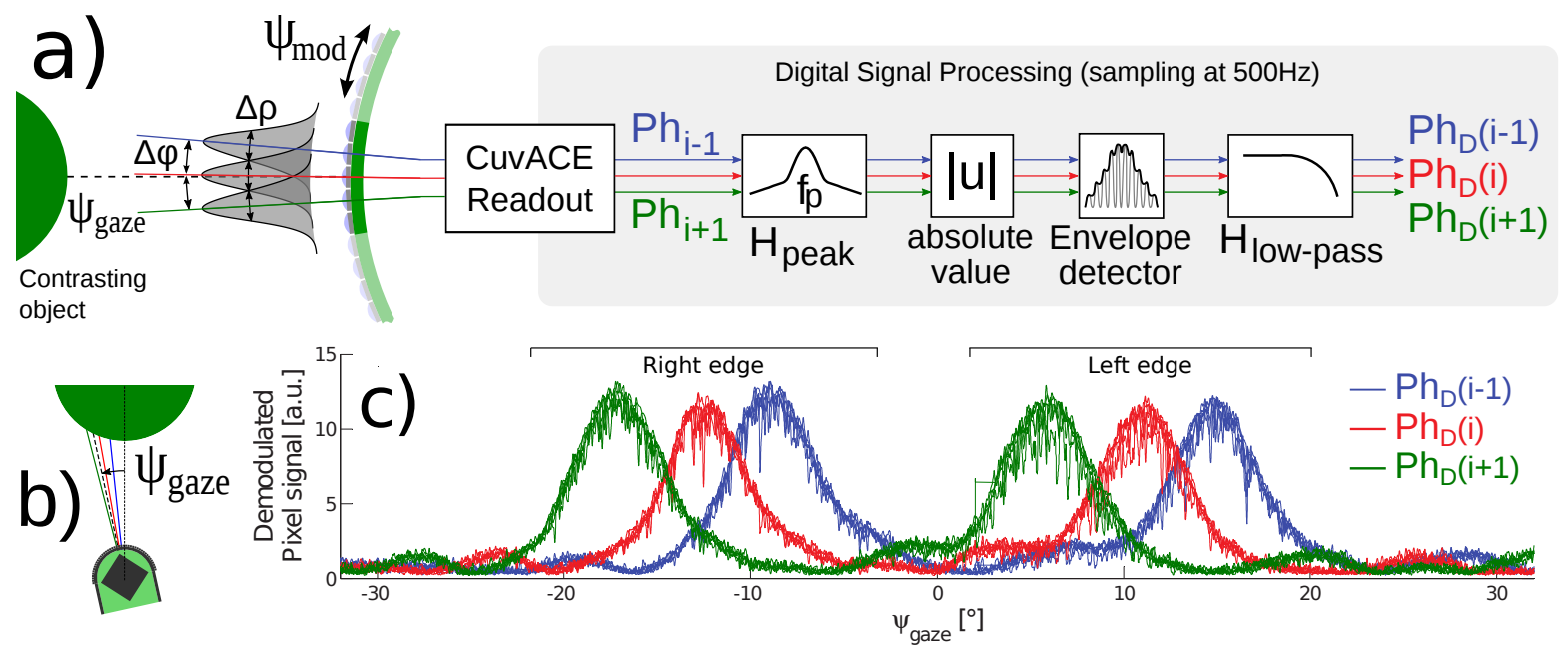

Figure 3. a) The demodulation signal processing algorithm applied to the visual signals delivered by the vibrating artificial compound eye $\left(\Psi_{\text {mod }}=A \sin (2 \pi 50 t)\right.$ where $A \approx \frac{\Delta \varphi}{2}$ ). Three Gaussian shaped optical angular sensitivities of the Active CurvACE (defined by its acceptance angle $\Delta \rho$ ) are shown. The CurvACE readout circuit is in charge of digitizing each pixel's output signal here at a sampling frequency of $500 \mathrm{~Hz}$. The subsequent digital demodulation steps are performed using a peak filter center at the scanning frequency cascaded with an absolute value function connected to a digital envelope detector and a low-pass filter (see Appendix B). b) Scheme of the Active CurvACE sensor in front of the target. c) Normalized Gaussian-like "Edge receptive fields" (defined by their standard deviation $\sigma$ ) of 3 vibrating photosensors after the demodulation process during two back and forth rotations of Active CurvACE (vibration turned $\mathrm{ON}$ ) in front of left and right edges of cylindrical target.

An adaptation of this principle was used here for the first time in the context of bio-inspired artificial vision. It was applied to a group of three in-line adjacent pixels (called a triplet) showing overlapped Gaussian-like responses of their "Edge receptive field" (see figure 3pc, and showed that once the WS has been normalized, this system produces a reliable output signal which can be used to locate an edge with great accuracy. Although biologically plausible, no founding proves that it is how the flies and more generally insects are processing the visual information. It is only a suggestion which in the context of the study is working reliably.

\subsubsection{Normalized Weighted Sum (NWS) Computation In the case of the present visual} system, the amplitude of the $P h_{D}$ signals depends on three main parameters: the ambient illuminance, the contrast and the scanning frequency. The novel feature of our approach is that it makes the visual sensor's output signal independent of these parameters by normalizing the $W S$ by the sum of the $P h_{D}$ signals. As shown in the Appendix C.2, the output of the normalized $W S\left(W S_{n o r m}=W S / S\right)$ is still linear and bounded. The hyperacuity of the $W S_{n o r m}$ was also established because the maximum linearity error amounts theoretically to less than $7 \%$ of the spacing between the receptive fields $\delta$. This makes it possible to measure the position of a contrasting edge. However, 
only one edge at a time must be present in the FOV of the photosensors used for the computations at a time. It is therefore best to use as few photosensors as possible. It was decided to use only three neighboring photosensors, i.e. one triplet, in the $W S_{\text {norm }}$, because when an edge is in the neighborhood of the center of one triplet's FOV $\left( \pm \frac{\delta}{2}\right)$, its 3 photosensors are contributing more than $99 \%$ to the total Sum.

To provide the pursuer with the angular position of one given edge, it is necessary to scale the $W S_{\text {norm }}$ value, depending on the spacing between each pixel. The mathematical expression of the normalized and scaled $W S$ (later noticed $N W S$ ) applied to an array of pixels featuring Gaussian angular sensitivities separated by an angle $\Delta \varphi$ can therefore be written as follows:

$$
N W S(i)=\Delta \varphi \cdot \frac{\sum_{k=i-1}^{i+1} k \cdot P h_{D}(k)}{\sum_{k=i-1}^{i+1} P h_{D}(k)}+b i a s
$$

where $P h_{D}$ denotes the photosensors' demodulated signals and bias is a calibrated offset used to set the zero position. Here, we intended to aligned the center of the cylinder with the center of the eye. Figure 4 a displays a schematic view of the computation from the pixel to the $N W S$ computation.
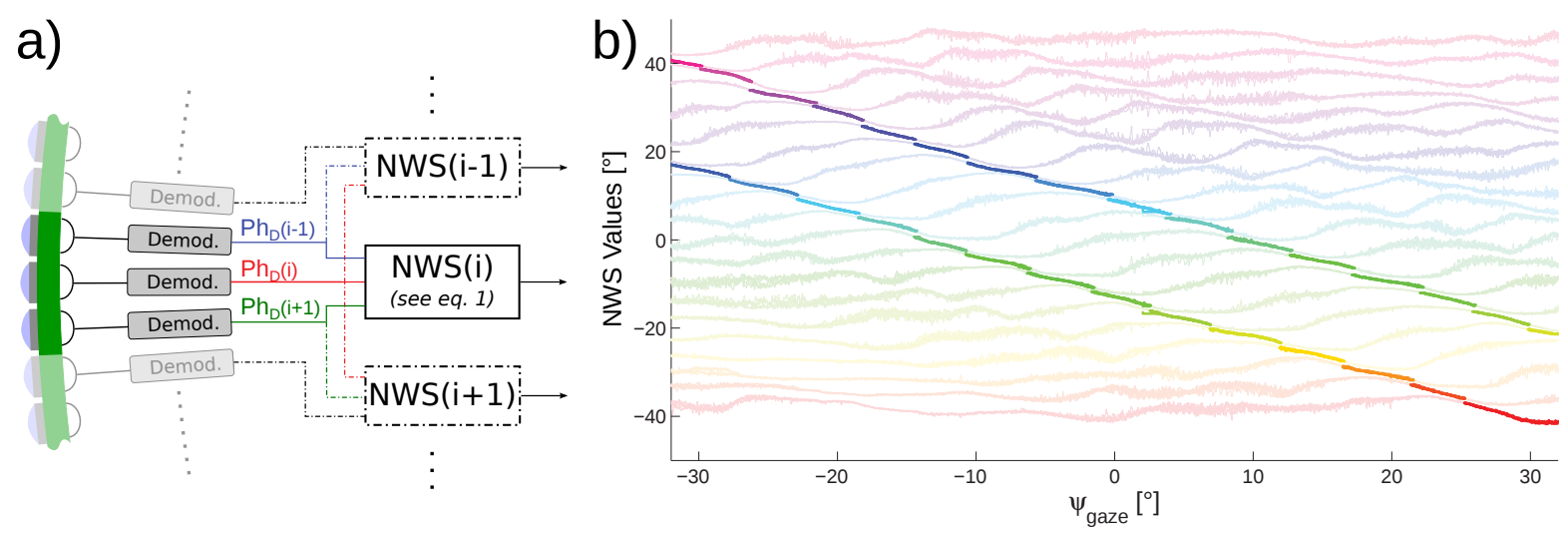

Figure 4. a) Layout of the computation of the Normalized and scaled $W S$. The $P h_{D}$ signals obtained after the demodulation process are the inputs of the different $N W S$ computations. b) $N W S$ values of the 21 triplets as a function of the visual sensor's gaze $\left(\psi_{\text {gaze }}\right)$ with respect to the fixed cylindrical target. The parts resulting from the selection process (see section 3.3) are highlighted.

3.2.3. Characterization of the vibrating visual sensor To test the responses of the hyperacute visual sensor (i.e., the Active CurvACE) depending on its orientation (denoted $\psi_{\text {gaze }}$ ) with respect to a textured cylindrical target, the visual sensor was turned towards a fixed target (a textured cylinder $30 \mathrm{~cm}$ in diameter, placed $91 \mathrm{~cm}$ ahead) and rotated stepwise about its vertical axis via an accessory position servo (not shown here) driven by a $0.1^{\circ}$ staircase signal, as in figure 3 . The responses of each of the 21 
$N W S$ were monitored at each azimuthal orientation of the gaze $\psi_{\text {gaze }}$ with respect to the target. The various $N W S$ were calculated by applying equation (1). Figure $4 \mathrm{~b}$ gives the results of all the $N W S$ calculations. With a theoretical uniform background, one can expect a constant response when no contrasts is present in the FOV. However, in a real scenario, contrast variations always appear as shadows or small changes in color. As the normalization makes the $N W S$ independent to contrast amplitude, it is difficult to identify the right contrast to track. Thus, a selection process is needed to obtain the $N W S$ of greatest interest, which will be used to locate the target.

\subsection{Weighted Sum Selection : edge localization over a large angular position range}

As shown in figure 4, a selection process was required to obtain the $N W S$ values corresponding to an edge. This process was performed using the following criteria:

$$
S_{\text {Criteria }}(i)=P h_{D}(i-1)+2 \cdot P h_{D}(i)+P h_{D}(i+1)
$$

The index $c_{j}$, giving the $N W S$ having the contrast $j$ in its FOV at time $t$, is then updated as follows:

$$
c_{j}(t)=\underset{i \in\left[c_{j}(t-1)-1, c_{j}(t-1)+1\right]}{\operatorname{argmax}} S_{\text {Criteria }}(i)
$$

This selection process was based on the assumption that the target is not moving faster than $\Delta \varphi$ between two successive sampling times. This means that an edge can only be located either via the same selected $N W S$ or via its nearest neighbor $\left(c_{j}(t)-1\right.$ or $\left.c_{j}(t)+1\right)$. In this study, the sampling frequency of the measurements was $500 \mathrm{~Hz}$.

The selection process can be applied to any number of edges to be followed. In the present case, as the target is a cylinder, two edges were tracked. Therefore, the edges positions are given as follows:

$$
\begin{aligned}
& \theta_{\text {Left }}=N W S\left(c_{\text {Left }}\right) \\
& \theta_{\text {Right }}=N W S\left(c_{\text {Right }}\right)
\end{aligned}
$$

Figure 5 summarizes the complete algorithm to get the edges localization and displays the comparisons of the measurements of the two edges compared to ground truth.

\subsection{Application to Target localization}

The two measured angular positions resulting from the selection process are denoted $\theta_{\text {Left }}$ and $\theta_{\text {Right }}$.

This algorithm makes it possible to locate two contrasting edges at the same time if they are separated by a sufficiently large angle, i.e. $2 \Delta \varphi$, in line with the hypothesis that only one contrast can be located at a time in the FOV of 3 photosensors. Assuming that these two edges belong to the same target, it is therefore possible to calculate its orientation $\epsilon_{r}$ and its subtended angle $\alpha$ defined as follows:

$$
\begin{aligned}
& \epsilon_{r}=\left(\theta_{\text {Left }}+\theta_{\text {Right }}\right) / 2 \\
& \alpha=\theta_{\text {Left }}-\theta_{\text {Right }}
\end{aligned}
$$


a)

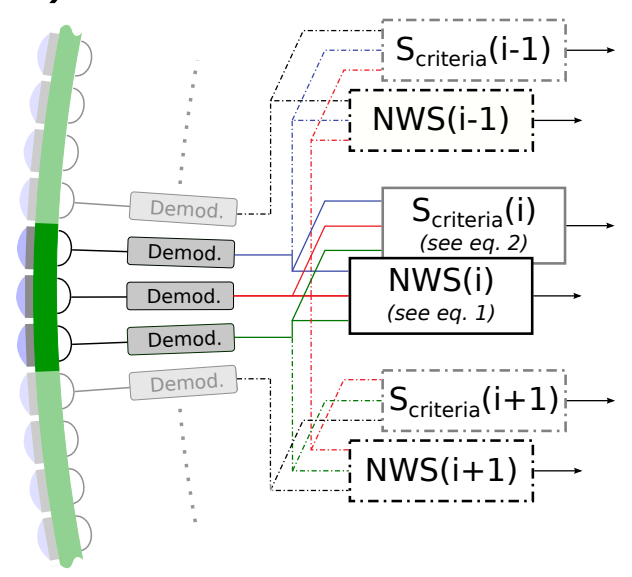

b)

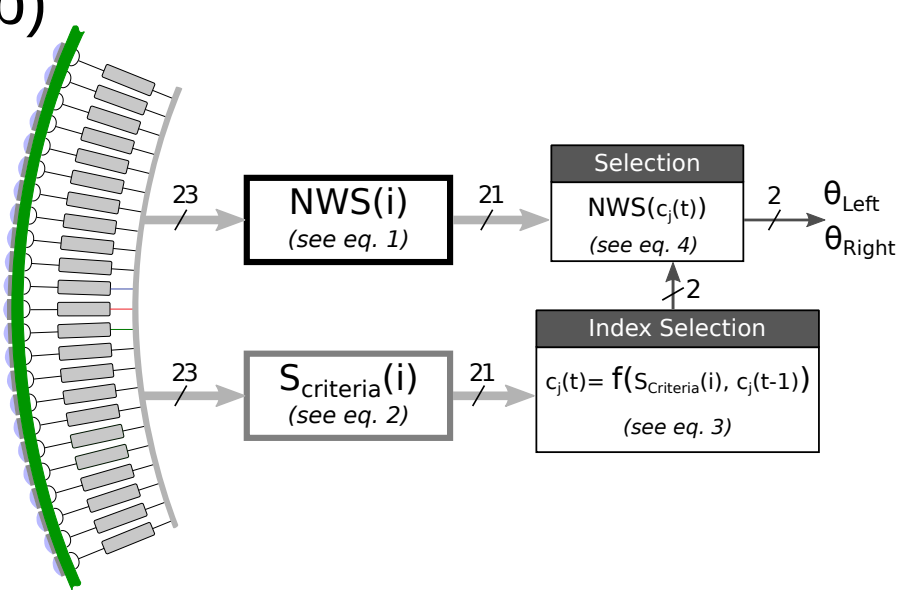

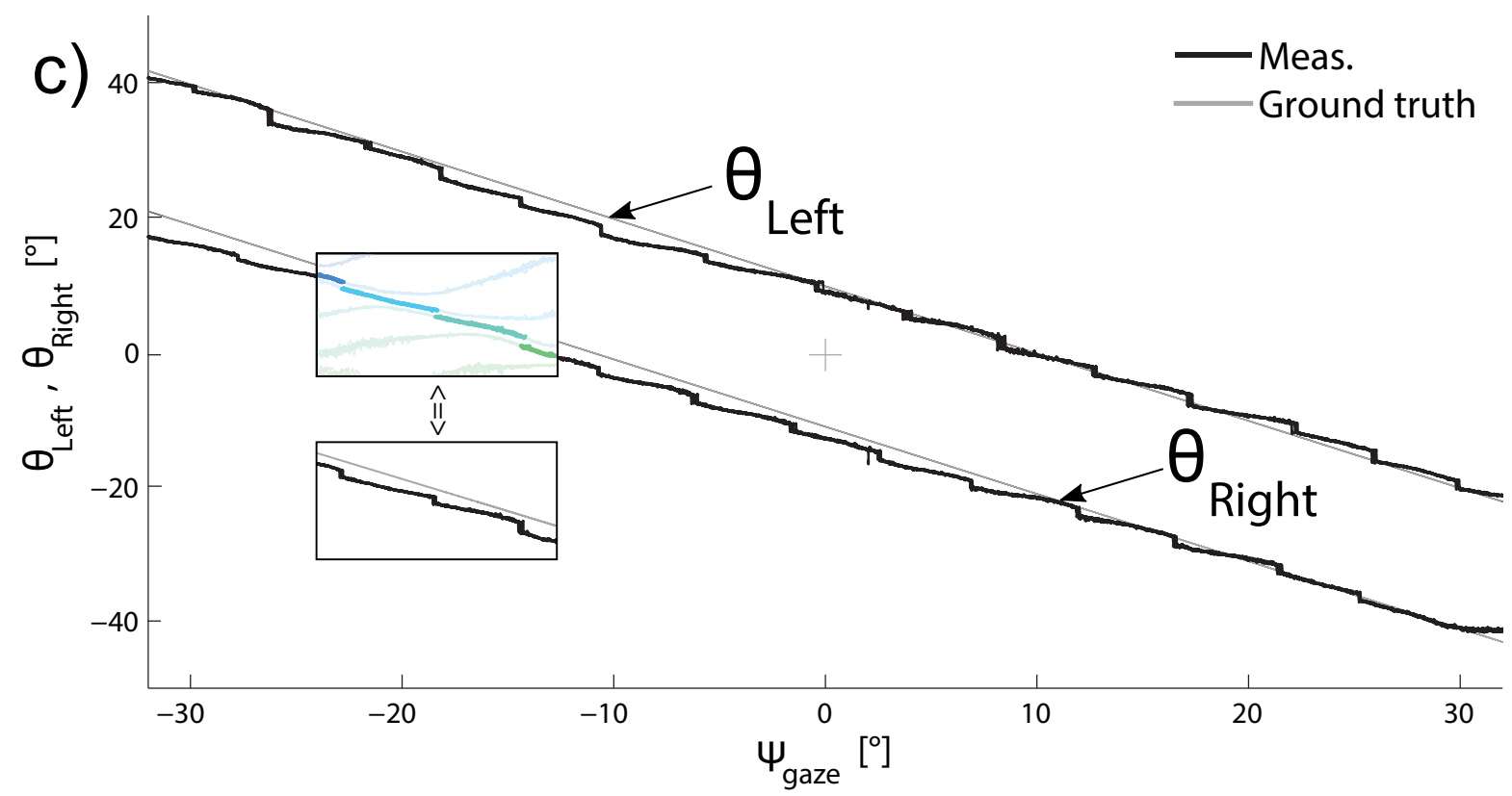

Figure 5. a) Layout of the $N W S$ computation and the $S_{\text {criteria }}$ b) Scheme of the computation from the pixel to the cylinder edges position measurement, through demodulation, $N W S$ processing and selection. c) Measured angular position of each target's edge (left and right) versus the visual sensor's gaze. Comparisons between the measured angular positions (red) and the ground truth values (green) based on a motion capture system showed that the linearity error of the sensor amounted to only 5 percent. The resolution (equivalent to 6 times of the signal standard deviation $\sigma$ ) was as small as $0.78^{\circ}$ with a signal-to-noise ratio of $20.7 \mathrm{~dB}$. The two frames highlights that the same results as in figure 40 are presented here differently.

Table 1 sums up the Active CurvACE performances.

\section{Nonlinear control and kinematics of the pursuer robot}

The artificial eye was placed on a robot equipped with Mecanum wheels (see Appendix E for details), which allows omnidirectional movements. The maximum speed that the 
robot was able to reach was equal to $0.5 \mathrm{~m} . \mathrm{s}^{-1}$.

The control laws implemented onboard the pursuer were drawn up using the kinematic model described previously by [49, 50]. The control strategy implemented was based on a non-linear approach to control each degree of freedom and a bounded control strategy adapted from [51] to control the 4-wheeled robot and also used in [52]. This controller takes in account the wheel speed limits, as it is impossible to go at full forward speed and rotate in the same time.

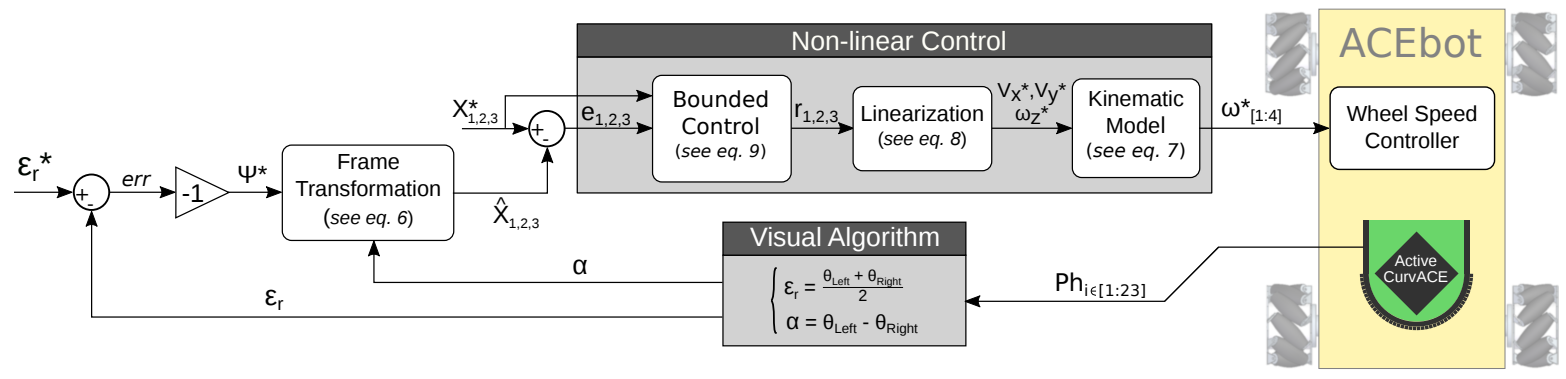

Figure 6. ACEbot non-linear controller scheme. The robot is using visual cues $\left(\epsilon_{r}, \alpha\right)$ to assess its orientation and distance to the target by controlling the 4 wheel speeds.

Figure 6 shows the ACEbot's control strategy. The setpoints $X^{*}$ are the desired position of the robot in the target frame. As the target is cylindrical, it has the same shape when viewed from all directions in the azimuthal plane. The robot's $x$ direction in the local target reference frame was therefore taken to be always co-linear with the pursuer-to-Target's direction, as shown in figure 1 b. Therefore, the $x$ reference is the distance requirement, which has to be negative. The $y$ reference in the target frame is null to ensure that ACEbot is aligned with the Target. It also means that no sideway movement will occur. The heading angle $\psi$ was controlled in the closed-loop mode so as to keep the retinal error $\epsilon_{r}$ equal to zero.

The estimated state vector can be expressed as follows:

$$
\hat{X}_{1,2,3}=\left[\begin{array}{c}
x_{r o b} \\
y_{r o b} \\
\psi_{r o b}
\end{array}\right]=\left[\begin{array}{c}
-\cos \left(\psi^{*}\right) \cdot\left(\frac{R_{\text {target }}}{\sin (\alpha / 2)}\right)-D_{\text {eye }} \\
0 \\
-\psi^{*}
\end{array}\right]
$$

where $D_{\text {eye }}$ is the distance from the eye to the ACEbot center. It can be noticed that $-x_{r o b}$ corresponds to the distance measurement (noted $D_{\text {meas }}$ in Table 1 ).

The kinematic model is the transfer matrix from the velocity of the robot's centre to the wheel speed:

$$
\left[\begin{array}{c}
\omega_{1}^{*} \\
\omega_{2}^{*} \\
\omega_{3}^{*} \\
\omega_{4}^{*}
\end{array}\right]=\frac{1}{R_{w}}\left[\begin{array}{ccc}
1 & -1 & -(l+L) \\
1 & 1 & (l+L) \\
1 & 1 & -(l+L) \\
1 & -1 & (l+L)
\end{array}\right]\left[\begin{array}{c}
v_{x}^{*} \\
v_{y}^{*} \\
\omega_{z}^{*}
\end{array}\right]
$$

where $v_{x}^{*}, v_{y}^{*}$ and $\omega_{z}^{*}$ are the robot speed control inputs. $R_{w}, L, l$ are the wheel radius, the wheelbase and the track width, respectively. The linearization is the transfer matrix 
from the global frame, or in this case, the Target, to the ACEbot frame, which is expressed as follows:

$$
\begin{aligned}
& v_{x}^{*}=\cos \left(\psi_{r o b}\right) \cdot r_{1}+\sin \left(\psi_{r o b}\right) \cdot r_{2} \\
& v_{y}^{*}=-\sin \left(\psi_{r o b}\right) \cdot r_{1}+\cos \left(\psi_{r o b}\right) \cdot r_{2} \\
& \omega_{z}^{*}=r_{3}
\end{aligned}
$$

where $r_{i}$ denotes the bounded control output signals. This transformation was also useful for decoupling the control of each degree of freedom in the global frame.

Considering the notation $e_{i}=X_{i}^{*}-\hat{X}_{i}$, the $r_{i}$ are the results of the following calculation:

$$
r_{i}=\sigma_{M_{i 3}}\left(\dot{X}_{i}^{*}+\sigma_{M_{i 2}}\left(a_{i 1} e_{i}+\sigma_{M_{i 1}}\left(a_{i 2} e_{i}+a_{i 1} a_{i 2} \int e_{i}\right)\right)\right)
$$

where the saturation function is defined as:

$$
\sigma_{M}(s)=\left\{\begin{array}{l}
s, \text { if }|s|<M \\
\operatorname{sign}(s) \cdot M,
\end{array} \quad\right. \text { otherwise }
$$

The values $a_{i j}$ and $M_{i j}$ are given in the Appendix D, It should be highlighted that this control strategy does not use any measured or estimated target speed as input. Therefore, there is no prediction on the future location of the target.

\section{Experimental pursuit performances}

\subsection{Experimental setup}

The experiments shows different pursuit trajectories. In all of them, the objective is to keep the retinal error $\epsilon_{r}$ to zero and a constant distance to the target, $0.7 \mathrm{~m}$ in sections 5.2 and 5.3 and $0.9 \mathrm{~m}$ in section 5.4 , respectively. The Target robot was controlled either by a manual remote control system or via a feedback loop, using a VICON Motion Capture System. This system can provide the position of the robots at a refresh rate of $500 \mathrm{~Hz}$ with a precision of less than $1.5 \mathrm{~mm}$ (see the Appendix B in [53]) and was used as the ground truth. ACEbot and the robotic Target were equipped with infrared (IR) LEDs used as active markers for the VICON system. The IR strobes of each VICON camera were disabled to prevent any visual perturbations from occurring due to the strong flickering infrared lighting.

\subsection{Ambient Light Variations}

Figure $7 \mathrm{a}$ shows the trajectory of the Target in green and that of the ACEbot in yellow. The positions of both robots are shown every $8 s$, and it can be seen that the ACEbot always kept close to the Target, while keeping it in its line of sight. The dark blue triangle shows the measured subtended angle.

In this experiment, the Target followed an imposed smooth trajectory with some small curves and ACEbot, after detecting the Target, was able to follow it unfailingly. 

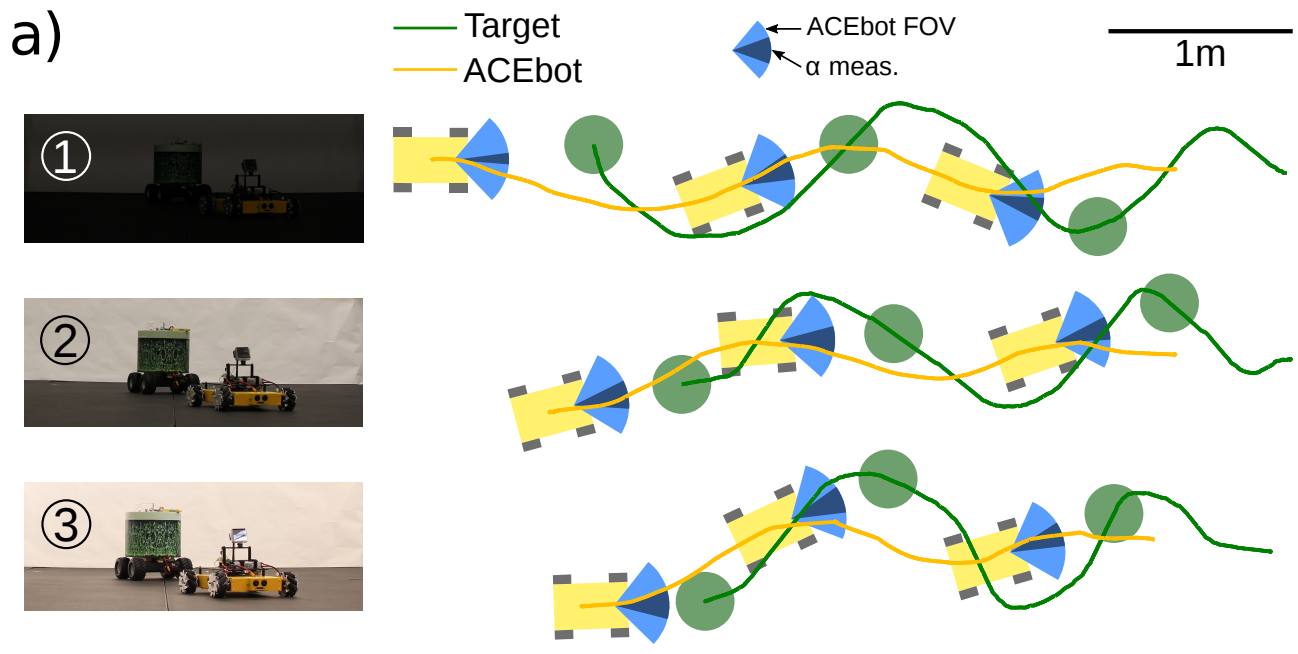

b)

(1)

(2)

(3)

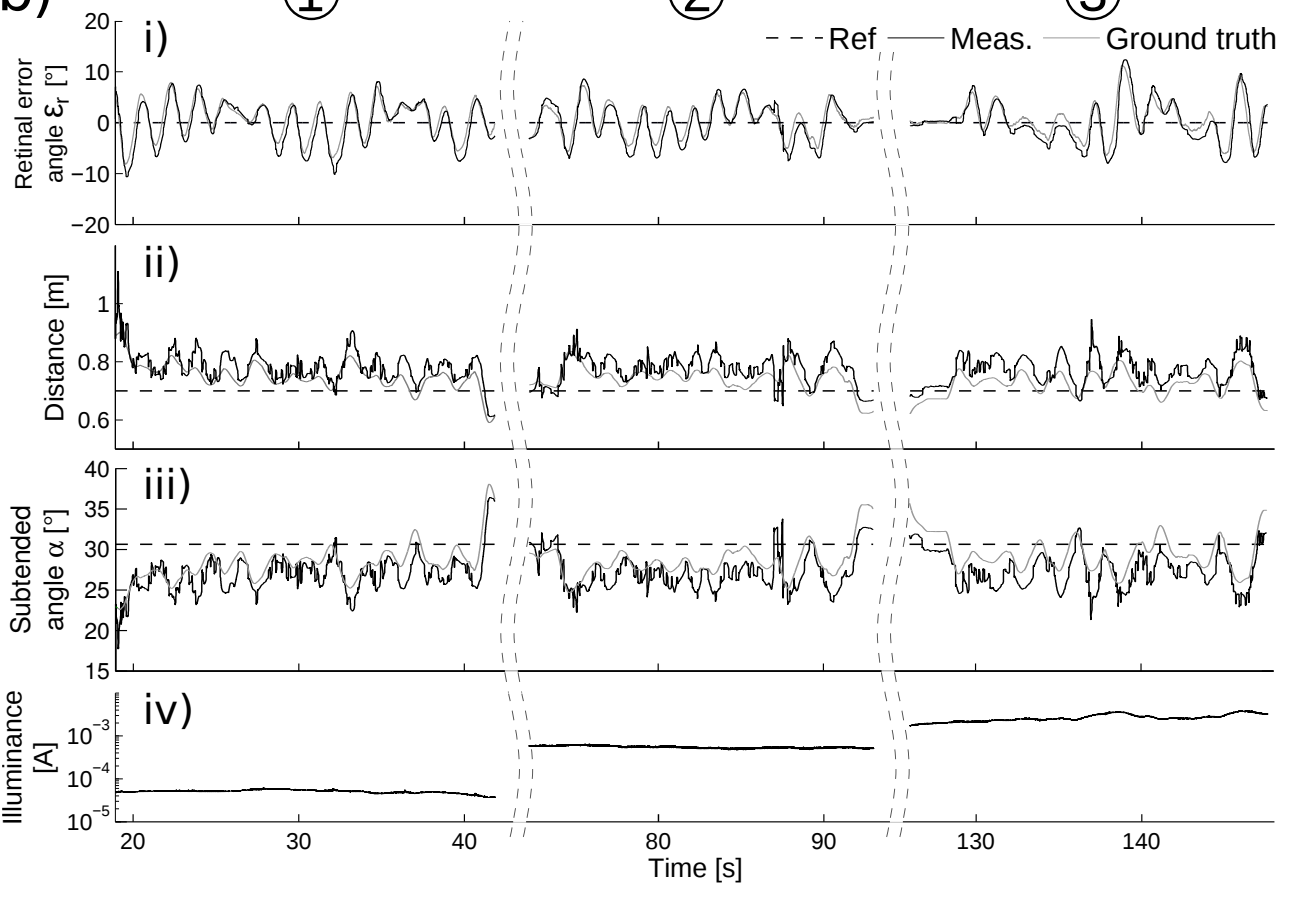

Figure 7. Three different following trajectories under different ambient light conditions. a) The trajectories of the center of inertia of the target and the pursuer ACEbot are presented in green and orange, respectively. The robots are drawn every $8 s$, with the full FOV of ACEbot in light blue, and the measured subtended angle of the target in dark blue. b-i) The retinal error $\epsilon_{r}$, i.e. position of the target in the FOV of ACEbot, with the setpoint, the measured angle and the ground truth in dashed black, black and grey, respectively. b-ii) depicts the distance from the target, and b-iii) the equivalent subtended angle in the FOV. The reference, the measurement and the ground truth are in dashed black, black and grey, respectively. b-iv) Dynamic responses of the light sensor shown in fig. 2a , reflecting the changes in the ambient lighting, which measured 100 Lux in (1), 780 Lux in (2) and 1500 Lux in (3). These responses show the robustness of the visual processing system with respect to several light levels. 
As the photosensors in the artificial eye adapted fast to changes in the light level [36], this pursuit behavior was consistently repeated under the various ambient lighting conditions tested. The tests of figure 7 (and also in the video in the supplementary data), were performed in a single experimental run, starting in the dark, where the target was detected and followed up to the end of the arena before returning close to the starting-point. The light was turned on when the target came to a stop. The pursuit was repeated and the blinds of the robotic arena were finally opened for the last pursuit. During this experiment, the target was remotely controlled by hand, giving similar trajectories.

It can be seen from figure 7 that the distance was accurately estimated and remained constant, although slightly above the reference value. This error was due to the fact that the ACEbot's speed is not large enough and no target speed is used in the control loop. The ACEbot's pursuit performances of the whole run are summarized in the table 1 .

\subsection{Repeatability}

To test the robustness of both the visual algorithm and the robot's control strategy, the target's and pursuer's trajectories were repeated 20 times under the same lighting conditions (fig. 8). It is worth noting that the pursuer took the same trajectory and never lost visual contact with the target. The standard deviations of both the target's and pursuer's trajectories were under $3 \mathrm{~cm}$.

\subsection{Biologically plausible behavior: chasing and motion camouflage}

5.4.1. Comparison with the hoverfly trajectories The strategy developed in this paper is based on the measurements of the angular position of two edges of the target, in

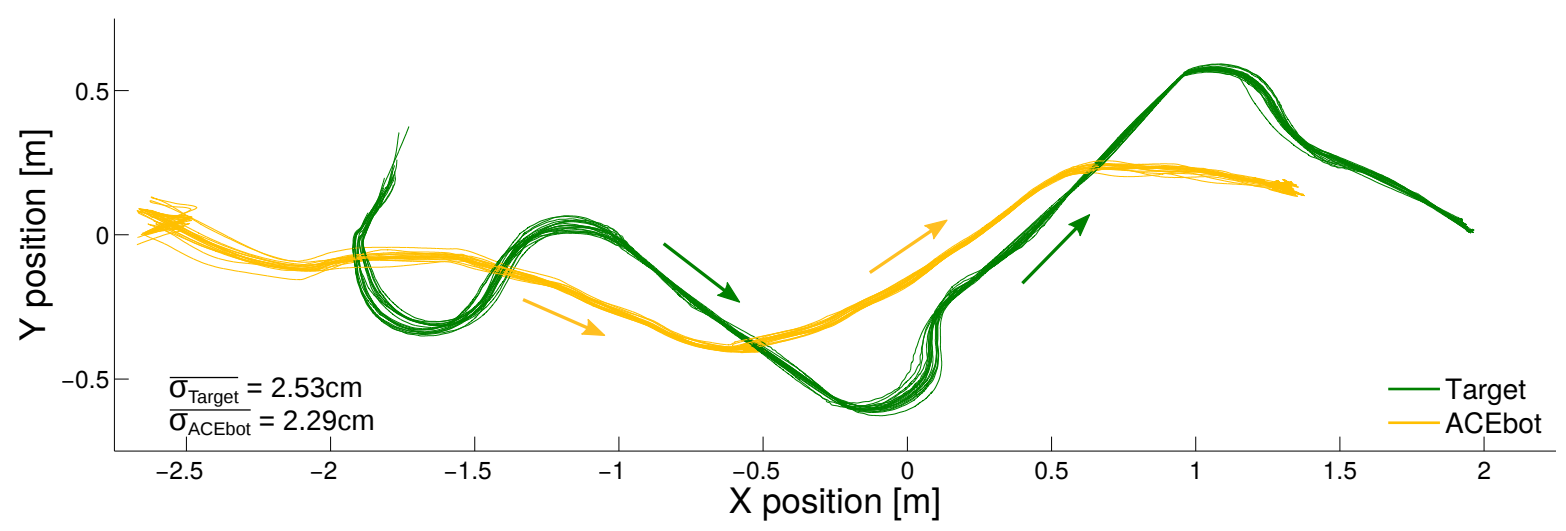

Figure 8. 20 recordings of target following tasks. The target's and pursuer's trajectories are plotted in green and yellow, respectively. This pursuing episode shows the excellent repeatability of the robot's ability to follow a target moving along several similar paths. The ACEbot consistently produced the same behavior, thus showing the high robustness of the visual processing algorithm. 
order to determine the subtended angle and the orientation of the target in the pursuer FOV. The measured subtended angle can be converted into a distance measurement on the assumption of a known target size. These measured orientation and distance are assessed so as to keep $\epsilon_{r}$ and $d$ equal to $0^{\circ}$ and $0.9 m$, respectively.

Collett and Land [3] observed some trajectories of the hoverfly Syritta Pipens $L$. that achieved a chasing behavior with a precision of $1 \mathrm{~cm}$ at a distance of $10 \mathrm{~cm}$. Similar trajectories between robotic and insect observations were achieved (see figure 9). Therefore, the strategy that consists of keeping the target in the middle of the FOV seems to be compatible with the biological observations. It should be highlighted
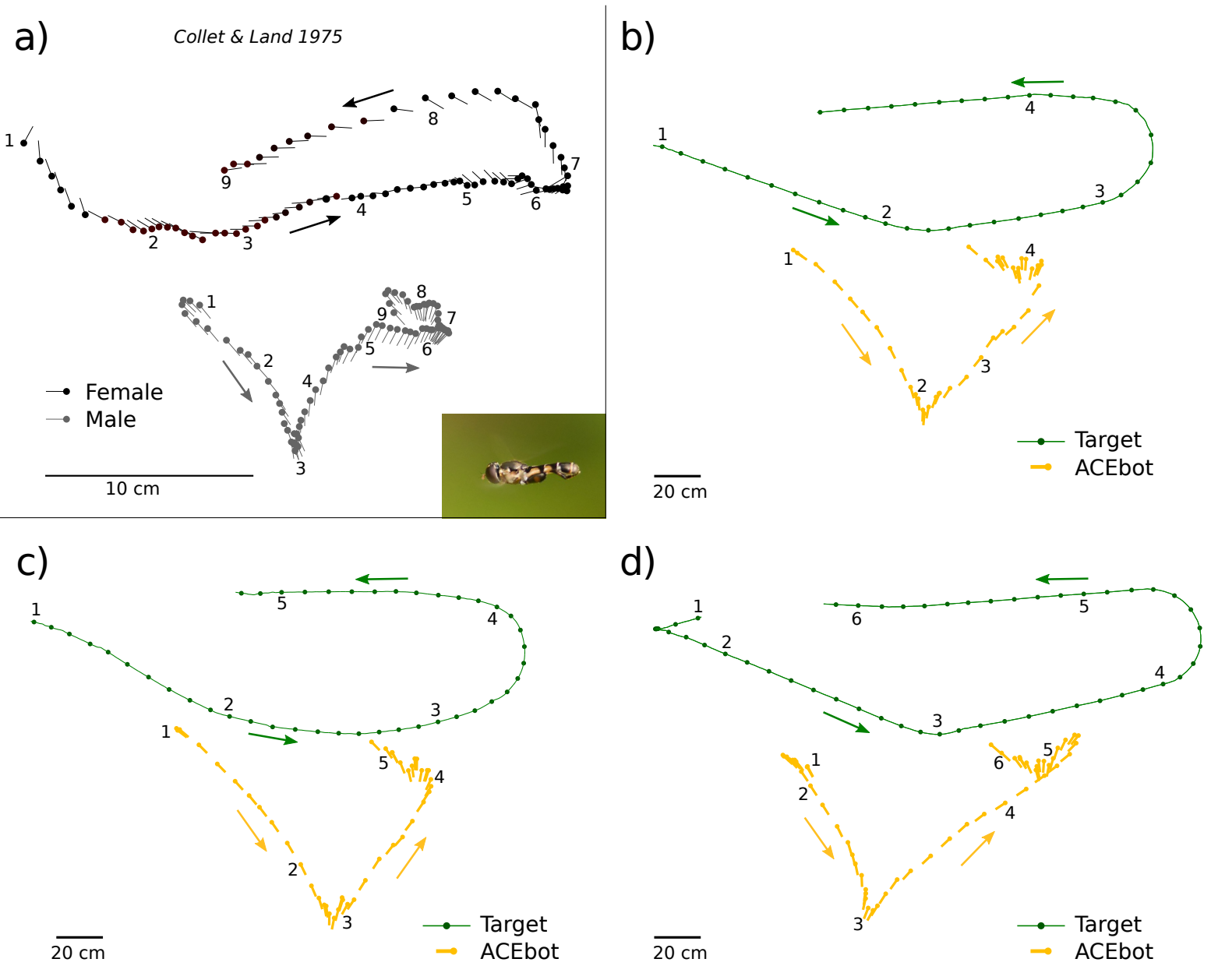

Figure 9. Comparison between the smooth pursuit observed during Syritta pipens L. mating process (a) and the target following trajectories of ACEbot (b, c and d). In a), the female and the male are represented in black and grey respectively. Dots are representing the head position and the lines are the orientation of the body. Each are spaced by $40 \mathrm{~ms}$ in time and the numbers indicate a $400 \mathrm{~ms}$ interval. Redrawn from [3. Hoverfly picture source wikimedia commons. In b-d), the target is represented by a green dot and a continuous line for the whole trajectory. ACEbot, the pursuer, is represented in yellow; the dot is the eye position and the line represents the robot orientation. The markers are separated by $400 \mathrm{~ms}$ and the numbers indicate a $4 \mathrm{~s}$ interval. 


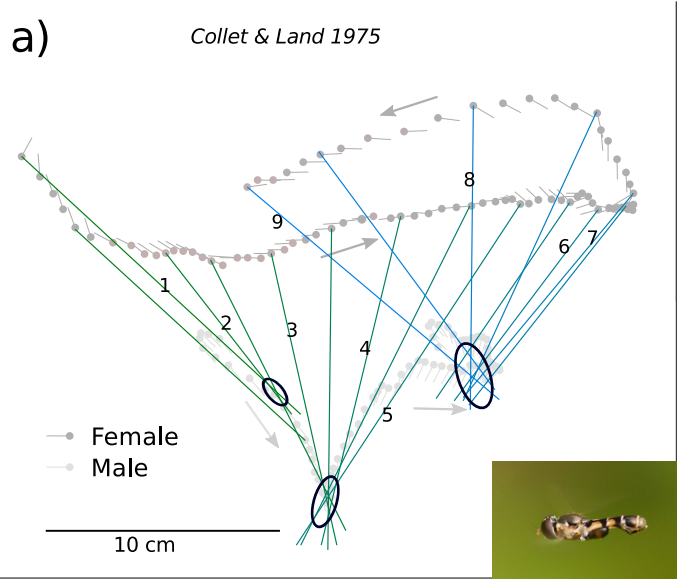

C)

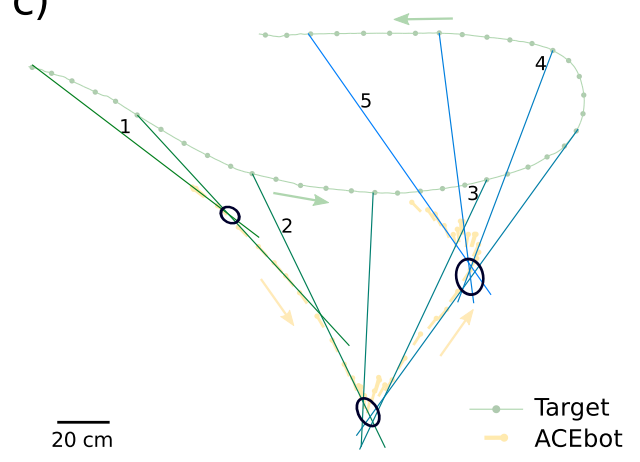

b)

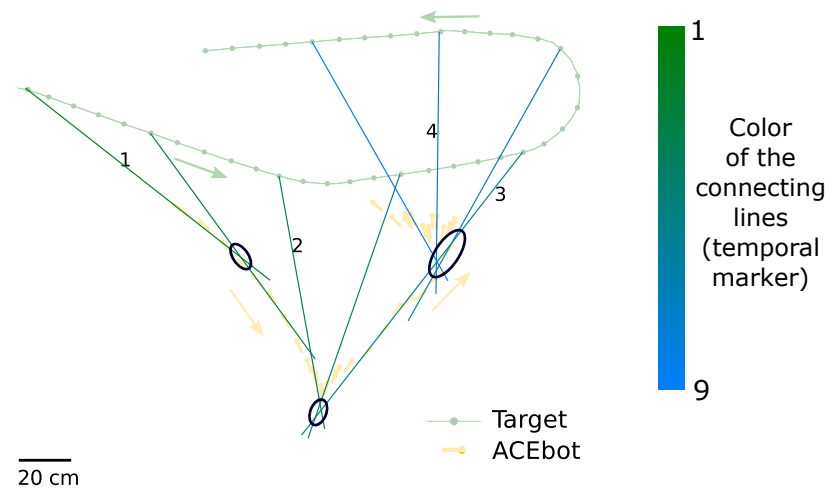

d)

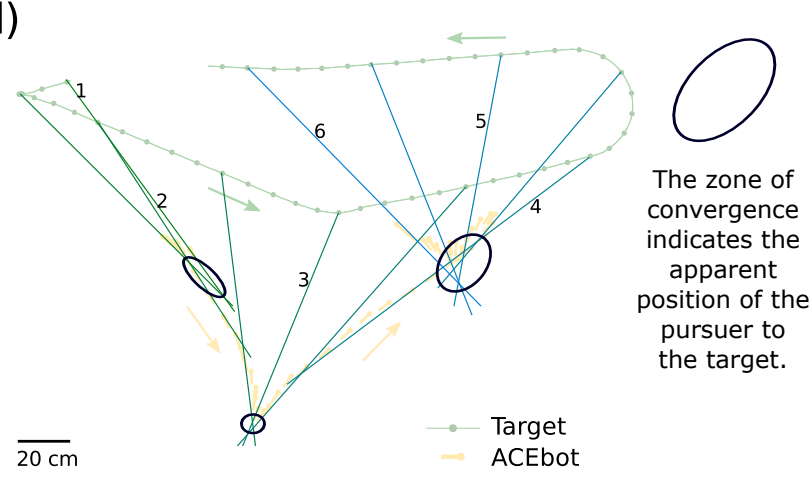

Figure 10. Connecting lines has been drawn between target-pursuer position on the trajectories presented in figure 9. Convergence zones of these connecting lines have been been highlighted. These zones of convergence indicates apparent static positions of the pursuer to the target. The convergence zones appear between large pursuer translation. The large radius of the convergence zone ellipses can be assessed to be below $10 \mathrm{~cm}$ in the robotic case.

that the insects are also using visual cues to maintain the distance from the mate. However, establishing a difference between a controller based on either the distance or the subtended angle measurement is difficult.

The Syritta pipiens $L$. seems to follow and maintain distance to its potential mate before trying to catch it using two different pursuit behaviors, possibly depending on the position of the mate in its field of view. It uses mostly continuous tracking when it is upfront but does body saccades and sway movements when the target is on the side. Sometimes, it was also observed that hoverfly does sideways movement even if the mate is in front of it, but these movements were unpredictable [3].

5.4.2. Discussion on motion camouflage In [30], the authors hypothesize that the male hoverfly follows the female while performing motion camouflage, i.e. assess its movement in order to appear as static onto the female retina, based on observations presented in [3]. One can notice that Collett and Land in their analysis did not suggested a motion camouflage behavior but only a pursuit at constant distance by keeping the target in the 
center of the FOV of the pursuer. In addition, only three motion camouflage trajectories were reported for the hoverfly Syritta Pipens L. (two in [30] and one here).

Figure 10 takes the same trajectories as in figure 9 with the addition of the directions linking the target to the pursuer for several positions. Three dense areas of successive connecting line crossings can be identified, similar for the insects and robots trajectories. One can say that a first stationary point is selected and changed during the pursuit to another one. For the hoverflies, it would have happened between time 2 and 3 and again between 5 and 6 (see figure 10a), where the areas are nearly aligned.

In this paper, we showed that a motion camouflage pattern can be observed when two simple rules are applied, keeping the target (i) in the center of the FOV of the pursuer by yaw rotation and (ii) at a constant distance (or constant subtended angle) by translation along the pursuer longitudinal axis (i.e. the pursuer-target direction).

Finally, with this simple control law, the resulting trajectory can create stationary points from the target perspective during the pursuit. Hence, is the pursuit of the male hoverfly the result of a specific strategy for motion camouflage or a consequence of a simpler vision-based strategy (as the one proposed in this paper)? More neuroethological researches should be done to be able to answer this question.

\section{Conclusion}

The Active CurvACE was used here as a biomimetic device to test successfully bioinspired strategy constrained by a bio-plausible visual sensor on a mobile robot. The high similarity in the robots and hoverflies trajectories as well as the biomimicry of the robotic visual system and of the summation processing provides support for the hypothesis that flies do maintain center the target and make its subtended angle constant during smooth pursuit. Indeed, the assessment of these two variables is sufficient to reproduce the hoverfly behavior. It is worth noting that the same sensorimotor control strategy was used in both cases: target following and fly-like smooth pursuit. The trajectories obtained were also compatible with motion camouflage.

On the robotic side, it was established here that only 23 pixels part of a vibrating artificial compound eye was able to locate a robotic target with hyperacuity and to pursue it with great accuracy. Compared to a previous study [44], we obtained a similar linearity in the target localization within a much larger visual range and without the need of a complex calibration process. The robot's orientation and its distance from the mobile target were precisely estimated under various lighting conditions. Table 1 gives the detailed results for the entire duration of the tests provided in the supplemental video. The novel computationally lean, small, lightweight bio-inspired visual sensor presented here is suitable for use in target following tasks.

The limitations of the present strategy concerned the existence of a sufficiently subtended angle of the Target in the FOV (theoretically $\geqslant 2 \Delta \varphi$ ) and the presence of a nearly uniform background or one without any sharp contrasts. An improvement of the pursuer dynamic responses could also be explored by providing the robot with 
Table 1. Active CurvACE and ACEbot performance summary

\begin{tabular}{|c|c|c|}
\hline \multicolumn{3}{|c|}{ Active CurvACE characteristics } \\
\hline \multirow{2}{*}{\multicolumn{3}{|c|}{ Static Optical parameters $\Delta \varphi=\Delta \rho=4.2^{\circ}$}} \\
\hline & & \\
\hline \multicolumn{3}{|c|}{ Scanning frequency $50 \mathrm{~Hz}$} \\
\hline \multicolumn{3}{|c|}{ Scanning angular amplitude $A \quad 2.1^{\circ}\left(\approx \frac{\Delta \varphi}{2}\right)$} \\
\hline \multicolumn{3}{|c|}{ Sampling frequency $500 \mathrm{~Hz}$} \\
\hline \multicolumn{3}{|c|}{ Visual processing bandwidth $20 \mathrm{~Hz}$} \\
\hline \multicolumn{3}{|c|}{ Hyperacuity resolution $0.78^{\circ}=$} \\
\hline \multicolumn{3}{|c|}{ Linearity } \\
\hline \multicolumn{3}{|c|}{ Signal to Noise Ratio $20.7 d B$} \\
\hline \multicolumn{3}{|c|}{$\begin{array}{l}\text { Estimated Target localization using Active CurvACE } \\
\text { with respect to the Ground Truth (GT) (mean } \pm \text { std }\end{array}$} \\
\hline \multicolumn{3}{|c|}{ Target ang. position $\epsilon_{r_{m e s}}-\epsilon_{r_{C T}}-0.62 \pm 1.28^{\circ}$} \\
\hline \multicolumn{3}{|c|}{ Subtended angle $\quad \alpha_{\text {meas }}-\alpha_{G T} \quad-1.78 \pm 1.49^{\circ}$} \\
\hline \multicolumn{3}{|c|}{ Target distance $\quad D_{\text {meas }}-D_{G T} \quad 3.1 \pm 2.7 \mathrm{~cm}$} \\
\hline \multicolumn{3}{|c|}{ Closed-loop ACEbot pursuit accuracy } \\
\hline Target ang. position & $\epsilon_{r}^{*}-\epsilon_{r_{G T}}$ & $-0.67 \pm 2.63^{\circ}$ \\
\hline Subtended angle & $\alpha_{R e f}-\alpha_{G T}$ & $-2.40 \pm 5.09^{\circ}$ \\
\hline Target distance & $D_{R e f}-D_{G T}$ & $2.8 \pm 7.9 \mathrm{~cm}$ \\
\hline
\end{tabular}

information about the target speed.

In the future, this active device could be embedded for example onboard a MicroAerial Vehicle (MAV). However, in the case of free flight, a second active Curvace or a 2D scanning would be required to detect the elevation of the target. In the automotive framework, a non-emissive optical sensor, like Active CurvACE, sensitive over a large angular position range and over a high range of light level could also provide a suitable alternative for the classical ultrasonic sensors and LIDARs which are being widely used these days in adaptive cruise control applications.

\section{Acknowledgments}

We thank M. Boyron and J. Diperi for assembling ACEbot, A. Manecy for designing the target robot's control, J.F. Guerrero-Castellanos for his advice on the robot's control and E. Loret for her help with the wheel speed control. This research was supported by CNRS (Life Science; Information Science; Engineering Science and Technology), AixMarseille University and the French National Research Agency (ANR) (IRIS project under ANR grants' number ANR-12-INSE-0009). 


\section{Appendix}

\section{Appendix A. Supplementary materials}

Videos of the experiences described in sections 5.2 and 5.4, can be found at the following links https://youtu.be/kdjJ6t7d2pM and https://youtu.be/ fciQr0o0G7g, respectively.

\section{Appendix B. Demodulation processing details}

The demodulation processing presented in figure 3 is the combination of different filters that need to be tuned, a peak filter, an envelope detector and a low pass filter. All of them are processed at a sampling frequency $f_{s}$ of $500 \mathrm{~Hz}$.

The peak filter is actually the combination of a second-order peak filter $H_{p f}$ and an equalizer filter $H_{e q}$. The peak filter has a peak frequency $f_{0}$ at $50 \mathrm{~Hz}$. Its z-transform is expressed as follows:

$$
H_{p f}(z)=(1-b) \cdot \frac{1-z^{-2}}{1-2 b \cos \left(\omega_{0}\right) z^{-1}+(2 b-1) z^{-2}}
$$

where $\omega_{o}=\frac{2 \pi f_{0}}{f_{s}}$ and $b=\frac{1}{1+\tan \left(10 \pi / f_{s}\right)}$

The z-transform of the equalizer filter is expressed as follows:

$$
\begin{aligned}
& H_{e q}(z)=\frac{(1+G \cdot \beta)-2 \cos \left(\omega_{0}\right) z^{-1}+(1-G \cdot \beta) z^{-2}}{1+\beta-2 \cos \left(\omega_{0}\right) z^{-1}+(1-\beta) z^{-2}} \\
& \beta=\sqrt{\frac{G_{B}^{2}-1}{G^{2}-G_{B}^{2}} \cdot \tan \left(\frac{20 \pi}{f_{s}}\right)}
\end{aligned}
$$

where $G=1.5$ and $G_{B}=1.155$.

The envelope detector reproduces digitally the behavior of an analogic envelop detector. It means that its output (out) is following the input signal (in) when this one is increasing. But it has an exponential decay when the input signal is decreasing. Mathematically, it is defined as follows:

out $(t)= \begin{cases}\text { out }(t-1)+\frac{T_{s}}{\tau}(\operatorname{in}(t)-\text { out }(t-1)) & \text { if out }(t-1)>\operatorname{in}(t) \\ \operatorname{in}(t) & \text { otherwise }\end{cases}$

where $T_{s}$ and $\tau$ are the sampling time and the time constant of the exponential decay, respectively. $\tau$ is chosen to be smaller than the modulation period, in our setup it is twice the period (i.e. $40 \mathrm{~ms}$ ).

Finally, the low-pass filter is simply a third-order low-pass filter with a cutoff frequency of $20 \mathrm{~Hz}$. 


\section{Appendix C. Details about the Heiligenberg Weighted Sum and the proposed normalization}

Appendix C.1. Theoretical presentation of the Normalized Weighted Sum

Given that the response of a Gaussian receptive field according to a stimulus position $x$ can be expressed as $e^{-\left(\frac{x-k}{\sigma}\right)^{2}}$, the equation proposed by Heiligenberg [47] reads as follows:

$$
W S(x, \sigma)=\sum_{k=-\infty}^{\infty} k \cdot e^{-\left(\frac{x-k}{\sigma}\right)^{2}}
$$

as $e^{-\left(\frac{x-k}{\sigma}\right)^{2}}$ describes a Gaussian function, where $\sigma$ is the standard deviation.

Let $S(x, \sigma)$, the sum of the Gaussian receptive fields, be defined as:

$$
S(x, \sigma)=\sum_{k=-\infty}^{\infty} e^{-\left(\frac{x-k}{\sigma}\right)^{2}}
$$

Figure C1 displays the responses of the $W S, S$ and $W S_{\text {norm }}$, which is equal to $W S / S$. It highlights that the Heiligenberg weighted sum linearity and its slope depend on the Gaussian overlapping. However, it can be seen that the normalization by the sum is helping, as it reduces the variation and ensures a constant slope whatever the width of the Gaussian.

Appendix C.2. Linear approximation and error estimation for the Normalized Weighted Sum computation

In order to bound the normalized weighted sum, we will apply a similar demonstration to the one used in [48]. Let $x=n+y$ with $n \in \mathbb{Z}$ and $y \in[0,1]$, which means that:

$$
W S(x, \sigma)=n \cdot S(y, \sigma)+W S(y, \sigma)
$$

where $W S$ and $S$ have been defined in equations (C.1) and (C.2), respectively.

It was established in [48, that:

$$
\begin{aligned}
& S(y, \sigma)=\sqrt{\pi} \sigma\left(1+2 \sum_{k=1}^{\infty} e^{-(\sigma k \pi)^{2}} \cos (2 \pi k y)\right) \\
& W S(y, \sigma)=y S(y, \sigma)-2 \pi^{\frac{3}{2}} \sigma^{3} \sum_{k=1}^{\infty} k e^{-(\sigma k \pi)^{2}} \sin (2 \pi k y)
\end{aligned}
$$

From the definition, $y=x-n$, which means that $S(x, \sigma)=S(y, \sigma)$ because $S$ is periodic with a period of 1 . The ratio of the weighted sum over the sum of the Gaussian functions is equal to $W S_{n o r m}=\frac{W S(x, \sigma)}{S(x, \sigma)}$. From that:

$$
\begin{aligned}
W S(x, \sigma)= & \sqrt{\pi} \sigma \cdot x\left(1+2 \sum_{k=1}^{\infty} e^{-\sigma^{2} k^{2} \pi^{2}} \cos (2 \pi k y)\right) \\
& -2 \pi \sigma^{2} \sqrt{\pi} \sigma \sum_{k=1}^{\infty} k \cdot e^{-\sigma^{2} k^{2} \pi^{2}} \sin (2 \pi k y)
\end{aligned}
$$


a)

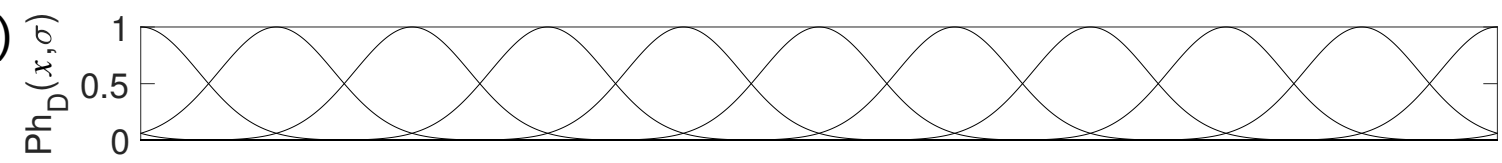

b) 200

$\sigma=0.25-\sigma=0.6 \quad-\cdot-\cdot \sigma=1 \quad \cdots \cdots \cdots \cdot \sigma=2$

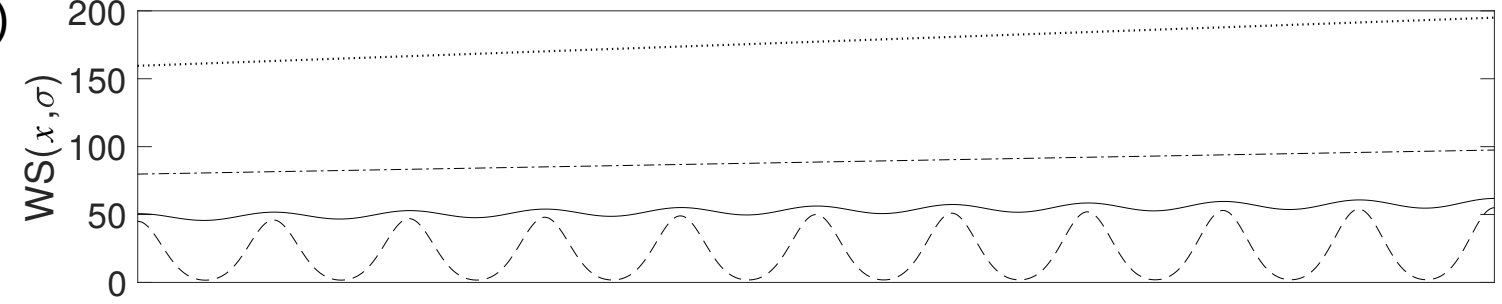

c)

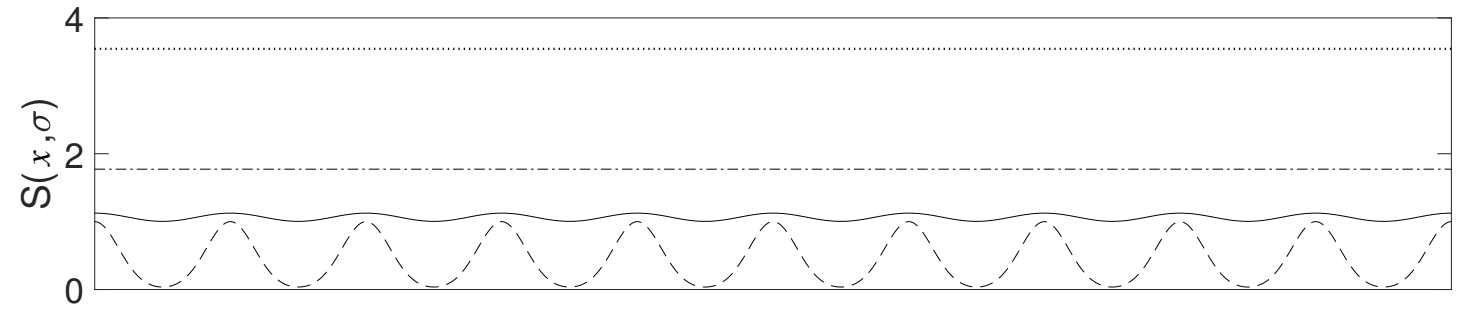

d)

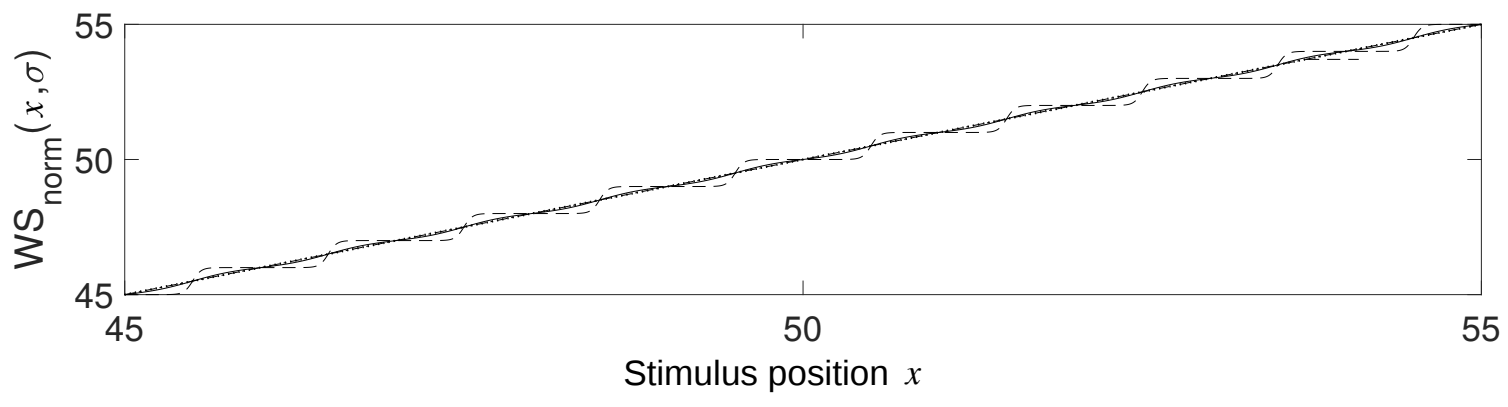

Figure C1. Presentation of the Weighted Sum and its normalization for perfectly Gaussian receptive fields. a) An array of Gaussian receptive field response spaced by an unitary space and with a half height width equal to one (i.e. $\sigma=1 /(2 \sqrt{\ln 2}) \approx 0.6)$. b-d) The theoretical Weighted Sum, the Sum and the normalized Weighted Sum $\left(W S_{\text {norm }}=W S / S\right)$ expressed for various standard deviation $\sigma$.

it can be deduced that,

$$
W S_{\text {norm }}=x-2 \pi \sigma^{2} \frac{\sum_{k=1}^{\infty} k \cdot e^{-\sigma^{2} k^{2} \pi^{2}} \sin (2 \pi k y)}{1+2 \sum_{k=1}^{\infty} e^{-\sigma^{2} k^{2} \pi^{2}} \cos (2 \pi k y)}
$$

The error $\epsilon$ (using $W S_{\text {norm }}=x+\epsilon$ ) can therefore be deduced as follows:

$$
\epsilon=-2 \pi \sigma^{2} \frac{\sum_{k=1}^{\infty} k \cdot e^{-\sigma^{2} k^{2} \pi^{2}} \sin (2 \pi k y)}{1+2 \sum_{k=1}^{\infty} e^{-\sigma^{2} k^{2} \pi^{2}} \cos (2 \pi k y)}
$$

If $\epsilon$ is bounded, we establish here that $W S_{n o r m}$ is similar to a linear function with a slope of 1 . 
From [48], crude bounds can be computed for the series, if the inequality $\frac{\sqrt{2 \ln 2}}{2 \pi} \leq \sigma$ is true:

$$
\begin{aligned}
& \left|\sum_{k=1}^{\infty} e^{-\sigma^{2} k^{2} \pi^{2}} \cos (2 \pi k y)\right| \leq \frac{e^{-\sigma^{2} \pi^{2}}}{1-e^{-\sigma^{2} \pi^{2}}} \\
& \left|\sum_{k=1}^{\infty} k \cdot e^{-\sigma^{2} k^{2} \pi^{2}} \sin (2 \pi k y)\right|<\frac{e^{-\sigma^{2} \pi^{2}}}{1-e^{-\sigma^{2} \pi^{2}}}
\end{aligned}
$$

Thus, the absolute value of $\epsilon$ can be bounded as follows:

$$
|\epsilon| \leq 2 \pi \sigma^{2} \frac{e^{-\sigma^{2} \pi^{2}}}{1-3 e^{-\sigma^{2} \pi^{2}}}
$$

In the present case, if we adopt the assumption that $\Delta \rho=\Delta \varphi, \sigma=\frac{1}{2 \sqrt{\ln (2)}}$, which satisfies the inequation $\frac{\sqrt{2 \ln 2}}{2 \pi} \leq \sigma$, the error $\epsilon$ will be equal to $7 \%$ of the spacing between each pair of photosensors. This value results from a crude mathematical error calculation, because one can obtain an error of $6.5 \%$ numerically with only 3 Gaussian functions.

\section{Appendix D. ACEbot control parameters}

The values for each control parameter are equal to: $a_{11}=22.5, a_{12}=0.0225, a_{21}=$ $22.5, a_{22}=0.0225, a_{32}=45.0, a_{33}=0$. These values make the trajectories very close to the linear region and guarantee the stability of the system. The limits $M_{i j}$ for $i, j=1,2,3$ are described in table D1.

Table D1. Saturation limits summary

\begin{tabular}{lll}
\hline$M_{11}=0.54$ & $M_{21}=0.675$ & $M_{31}=0.9360$ \\
$M_{12}=2.5515$ & $M_{22}=2.835$ & $M_{32}=5.256$ \\
$M_{13}=7.0425$ & $M_{23}=7.0425$ & $M_{33}=1.5$ \\
\hline
\end{tabular}

\section{Appendix E. Information about the Robotic platform ACEbot}

The product reference of the platform is "Nexus 4WD Mecanum wheel mobile robot kit 10015 " but the ultrasonic sensors are not used in the present application.

As regards the electronics, the main program was running on a Gumstix Overo board, which, thanks to the RT-MaG toolbox, can be programmed directly from Matlab/Simulink [54]. This Linux based Computer-On-Module (COM) was running the signal processing algorithm for the 23 photosensors received from CurvACE through a synchronous serial bus (SPI). It was also connected through UART to a Teensy board. The latter was in charge of the acquisition of the photo-current sensor to record the ambient light level and to switch the stepper motor in charge of the eye's periodic tremor 


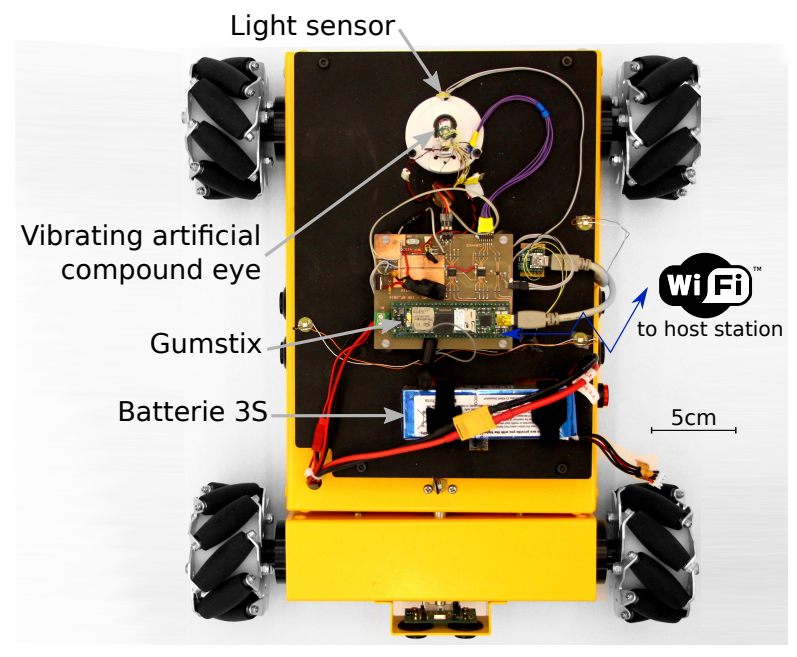

Figure E1. Picture from above of the robot, showing the electronic hardware. The Gumstix communicates with the ground station through Wifi as well as with the Motor control board and with the Teensy through an UART bus. Neither boards are visible on the picture as they are under the robot.

on and off. The wheel speed setpoints computed on the Gumstix were transmitted through a serial bus (UART) to the Arduino-compatible motor control board, which then controlled each wheel's speed with a PI controller. A WiFi connection between the robot and the ground station was used to monitor the variables of interest during the experiments and provided the rover with setpoints. The ACEbot robot was powered by a 3-cell lithium polymer battery.

\section{Bibliography}

[1] M.F. Land. Visual tracking and pursuit: Humans and arthropods compared. Journal of Insect Physiology, 38(12):939 - 951, 1992.

[2] M.F. Land and T.S. Collett. Chasing behaviour of houseflies (fannia canicularis). Journal of Comparative Physiology, 89(4):331-357, 1974.

[3] T.S. Collett and M.F. Land. Visual control of flight behaviour in the hoverfly syritta pipiens 1. Journal of Comparative Physiology, 99(1):1-66, 1975.

[4] N. Boeddeker, R. Kern, and M. Egelhaaf. Chasing a dummy target: smooth pursuit and velocity control in male blowflies. Proceedings of the Royal Society of London B: Biological Sciences, 270(1513):393-399, 2003.

[5] T.J. Wardill, S.T. Fabian, A.C. Pettigrew, D.G. Stavenga, K. Nordström, and P.T. GonzalezBellido. A novel interception strategy in a miniature robber fly with extreme visual acuity. Current Biology, 27(6):854 - 859, 2017.

[6] T. S. Collett and M. F. Land. How hoverflies compute interception courses. Journal of comparative physiology, 125(3):191-204, 1978.

[7] R.M. Olberg, A.H. Worthington, and K.R. Venator. Prey pursuit and interception in dragonflies. Journal of Comparative Physiology A, 186(2):155-162, 2000.

[8] J.R. Dunbier, S.D. Wiederman, and D.C. O'Carroll. Predictive response facilitation to moving targets in an insect neuron. In 10th International Congress of Neuroethology, number 234, 2012. 
[9] K. Nordström and D.C. O'Carroll. Feature detection and the hypercomplex property in insects. Trends in Neurosciences, 32(7):383 - 391, 2009.

[10] K. Nordström. Neural specializations for small target detection in insects. Current Opinion in Neurobiology, 22(2):272 - 278, 2012. Neuroethology.

[11] Z.M. Bagheri, S.D. Wiederman, B.S. Cazzolato, S. Grainger, and D.C. OCarroll. Performance of an insect-inspired target tracker in natural conditions. Bioinspiration $\mathscr{E}$ Biomimetics, 12(2):025006, 2017.

[12] Y. Wu, J. Lim, and M. H. Yang. Online object tracking: A benchmark. In Computer Vision and Pattern Recognition (CVPR), 2013 IEEE Conferenceon, pages 2411-2418, June 2013.

[13] Z.M. Bagheri, B.S. Cazzolato, S. Grainger, D.C. O'Carroll, and S.D. Wiederman. An autonomous robot inspired by insect neurophysiology pursues moving features in natural environments. Journal of Neural Engineering, 14(4):046030, 2017.

[14] H. Liu, D. P. Moeys, G. Das, D. Neil, S. C. Liu, and T. Delbruck. Combined frame- and eventbased detection and tracking. In 2016 IEEE International Symposium on Circuits and Systems (ISCAS), pages 2511-2514, May 2016.

[15] G. L. Mariottini, F. Morbidi, D. Prattichizzo, N. Vander Valk, N. Michael, G. Pappas, and K. Daniilidis. Vision-based localization for leader-follower formation control. IEEE Transactions on Robotics, 25(6):1431-1438, Dec 2009.

[16] A. K. Das, R. Fierro, V. Kumar, J. P. Ostrowski, J. Spletzer, and C. J. Taylor. A vision-based formation control framework. IEEE Transactions on Robotics and Automation, 18(5):813-825, Oct 2002 .

[17] N. Cowan, O. Shakerina, R. Vidal, and S. Sastry. Vision-based follow-the-leader. In Intelligent Robots and Systems, 2003.(IROS 2003). Proceedings. 2003 IEEE/RSJ International Conference on, volume 2, pages 1796-1801. IEEE, 2003.

[18] J.F. Roberts, T. Stirling, J.-C. Zufferey, and D. Floreano. 3-d relative positioning sensor for indoor flying robots. Autonomous Robots, 33(1):5-20, 2012.

[19] K.E. Wenzel, A. Masselli, and A. Zell. Visual tracking and following of a quadrocopter by another quadrocopter. In Intelligent Robots and Systems (IROS), 2012 IEEE/RSJ International Conference on, pages 4993-4998. IEEE, 2012.

[20] M. Faessler, E. Mueggler, K. Schwabe, and D. Scaramuzza. A monocular pose estimation system based on infrared leds. In 2014 IEEE International Conference on Robotics and Automation (ICRA), pages 907-913, May 2014.

[21] D. Shishika, J.K. Yim, and D.A. Paley. Bio-inspired pursuit with autonomous hovercraft using lyapunov-based control. In American Control Conference (ACC), 2015, pages 3107-3113. IEEE, July 2015.

[22] M. Saska. Mav-swarms: Unmanned aerial vehicles stabilized along a given path using onboard relative localization. In Unmanned Aircraft Systems (ICUAS), 2015 International Conference on, pages 894-903, June 2015.

[23] M. Nitsche, T. Krajnik, P. Cizek, M. Mejail, T. Duckett, et al. Whycon: an efficient, markerbased localization system. In Workshop on Aerial Open-source Robotics, 28 September 2015, Hamburg, 2015.

[24] M. Basiri, F. Schill, P. Lima, and D. Floreano. On-board relative bearing estimation for teams of drones using sound. IEEE Robotics and Automation Letters, 1(2):820-827, July 2016.

[25] P.M. Maxim, S. Hettiarachchi, W.M. Spears, D.F. Spears, J. Hamann, T. Kunkel, and C. Speiser. Trilateration localization for multi-robot teams. In in Proceedings of the Sixth International Conference on Informatics in Control, Automation and Robotics, Special Session on MultiAgent Robotic Systems (ICINCO'08, 2008.

[26] R. Strydom, S. Thurrowgood, A. Denuelle, and M.V. Srinivasan. Uav guidance: A stereobased technique for interception of stationary or moving targets. In Clare Dixon and Karl Tuyls, editors, Towards Autonomous Robotic Systems, pages 258-269, Cham, 2015. Springer International Publishing. 
[27] N.A. Shneydor. Missile guidance and pursuit: kinematics, dynamics and control. Elsevier, 1998.

[28] S.H. Lim, T. Furukawa, G. Dissanayake, and H. F. Durrant-Whyte. A time-optimal control strategy for pursuit-evasion games problems. In IEEE International Conference on Robotics and Automation, 2004. Proceedings. ICRA '04. 2004, volume 4, pages 3962-3967 Vol.4, April 2004.

[29] N. An, S. Sun, X. Zhao, and Z. Hou. Move like humans: End-to-end gaussian process regression based target tracking control for mobile robots. In 2017 36th Chinese Control Conference (CCC), pages 6917-6921, July 2017.

[30] M.V. Srinivasan and M. Davey. Strategies for active camouflage of motion. Proceedings of the Royal Society of London B: Biological Sciences, 259(1354):19-25, 1995.

[31] M.V. Srinivasan A. Mizutani, J.S. Chahl. Motion camouflage in dragonflies. Nature, 423:604, Jun 2003.

[32] A.J. Anderson and P.W. McOwan. Model of a predatory stealth behaviour camouflaging motion. Proceedings of the Royal Society of London B: Biological Sciences, 270(1514):489-495, 2003.

[33] E.W Justh and P.S Krishnaprasad. Steering laws for motion camouflage. Proceedings of the Royal Society of London A: Mathematical, Physical and Engineering Sciences, 462(2076):3629-3643, 2006.

[34] P. V. Reddy, E. W. Justh, and P. S. Krishnaprasad. Motion camouflage with sensorimotor delay. In 2007 46th IEEE Conference on Decision and Control, pages 1660-1665, Dec 2007.

[35] R. Strydom and M.V. Srinivasan. Uas stealth: target pursuit at constant distance using a bioinspired motion camouflage guidance law. Bioinspiration \&3 Biomimetics, 12(5):055002, 2017.

[36] D. Floreano, R. Pericet-Camara, S. Viollet, F. Ruffier, A. Brückner, R. Leitel, W. Buss, M. Menouni, F. Expert, R. Juston, M.K. Dobrzynski, G. L'Eplattenier, F. Recktenwald, H.A. Mallot, and N. Franceschini. Miniature curved artificial compound eyes. Proc Natl Acad Sci U $S$ A, 110(23):9267-9272, Jun 2013.

[37] S. Viollet, S. Godiot, R. Leitel, W. Buss, P. Breugnon, M. Menouni, R. Juston, F. Expert, F. Colonnier, G. L'Eplattenier, A. Brückner, F. Kraze, H. Mallot, N. Franceschini, R. PericetCamara, F. Ruffier, and Dario Floreano. Hardware architecture and cutting-edge assembly process of a tiny curved compound eye. Sensors, 14(11):21702, 2014.

[38] S. Viollet. Vibrating makes for better seeing: from the fly's micro eye movements to hyperacute visual sensors. Frontiers in Bioengineering and Biotechnology, 2(9), 2014.

[39] P.N. Prokopowicz and P.R. Cooper. The dynamic retina: Contrast and motion detection for active vision. International Journal of Computer Vision, 16(3):191-204, 1995.

[40] M. O. Hongler, Y. L. de Meneses, A. Beyeler, and J. Jacot. The resonant retina: exploiting vibration noise to optimally detect edges in an image. IEEE Transactions on Pattern Analysis and Machine Intelligence, 25(9):1051-1062, Sept 2003.

[41] F. Mura and I. Shimoyama. Visual guidance of a small mobile robot using active, biologicallyinspired, eye movements. In Robotics and Automation, 1998. Proceedings. 1998 IEEE International Conference on, volume 3, pages 1859-1864, 16-20 May 1998.

[42] G. Westheimer. Visual hyperacuity. Sensory Physiology 1. Springer-Verlag, 1981.

[43] L. Kerhuel, S. Viollet, and N. Franceschini. The vodka sensor: A bio-inspired hyperacute optical position sensing device. Sensors Journal, IEEE, 12(2):315-324, 2012.

[44] A. Manecy, J. Diperi, M. Boyron, N. Marchand, and S. Viollet. Novel hyperacute gimbal eye for implementing precise hovering and target tracking on board a quadrotor. In IEEE International Conference on Robotics and Automation (ICRA), 2016.

[45] F. Colonnier, A. Manecy, R. Juston, H. Mallot, R. Leitel, D. Floreano, and S. Viollet. A smallscale hyperacute compound eye featuring active eye tremor: application to visual stabilization, target tracking, and short-range odometry. Bioinspiration $\mathcal{E}$ Biomimetics, 10(2):026002, 2015.

[46] R. Juston and S. Viollet. A miniature bio-inspired position sensing device for the control of micro-aerial robots. In Intelligent Robots and Systems (IROS), 2012 IEEE/RSJ International Conference on, pages 1118-1124, Oct 2012. 
[47] W. Heiligenberg. Central processing of sensory information in electric fish. Journal of Comparative Physiology A, 161(4):621-631, 1987.

[48] P Baldi and W Heiligenberg. How sensory maps could enhance resolution through ordered arrangements of broadly tuned receivers. Biological cybernetics, 59(4-5):313-318, 1988.

[49] C.-C. Tsai, F.-C. Tai, and Y.-R. Lee. Motion controller design and embedded realization for mecanum wheeled omnidirectional robots. In Proceedings of the 8th World Congress on Intelligent Control and Automation, pages 546-551, June 2011.

[50] P. Viboonchaicheep, A. Shimada, and Y. Kosaka. Position rectification control for mecanum wheeled omni-directional vehicles. Industrial Electronics Society, 2003. IECON '03. The 29th Annual Conference of the IEEE, 1:854-859, Nov. 2003.

[51] J.F. Guerrero-Castellanos, M.G. Villarreal-Cervantes, J.P. Sánchez-Santana, and S. RamírezMartínez. Trajectory tracking of a mobile robot $(3,0)$ by means of bounded control. Revista Iberoamericana de Automática e Informática Industrial \{RIAI\}, 11(4):426 - 434, 2014.

[52] T. Raharijaona, R. Mawonou, T.V. Nguyen, F. Colonnier, M. Boyron, J. Diperi, and S. Viollet. Local positioning system using flickering infrared leds. Sensors, 17(2518), 2017.

[53] A. Manecy, N. Marchand, F. Ruffier, and S. Viollet. X4-mag: A low-cost open-source microquadrotor and its linux-based controller. International Journal of Micro Air Vehicles, 7(2):89$110,2015$.

[54] A. Manecy. RT-MaG project. http://www.gipsa-lap.fr/projet/RT-MaG/. 